\title{
Computerized Clinical Decision Support System for Emergency Department-Initiated Buprenorphine for Opioid Use Disorder: User-Centered Design
}

Jessica M Ray ${ }^{1}, \mathrm{PhD}$; Osama M Ahmed ${ }^{1}$, BSc; Yauheni Solad ${ }^{2}, \mathrm{MD}$; Matthew Maleska ${ }^{3}$, MBA; Shara Martel ${ }^{1}$, MPH, MS; Molly M Jeffery ${ }^{4}$, PhD; Timothy F Platts-Mills ${ }^{5}$, MSc, MD; Erik P Hess ${ }^{6}$, MSc, MD; Gail D’ Onofrio ${ }^{1}$, MD, MS; Edward R Melnick ${ }^{1}$, MD, MHS

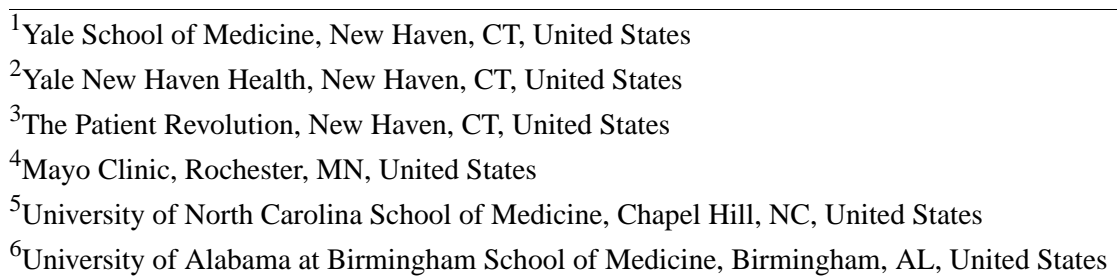

Corresponding Author:

Edward R Melnick, MD, MHS

Yale School of Medicine

464 Congress Ave, Suite 260

New Haven, CT, 06519

United States

Phone: 19176081760

Fax: 12037854580

Email: edward.melnick@yale.edu

\section{Abstract}

Background: Emergency departments (EDs) frequently care for individuals with opioid use disorder (OUD). Buprenorphine (BUP) is an effective treatment option for patients with OUD that can safely be initiated in the ED. At present, BUP is rarely initiated as a part of routine ED care. Clinical decision support (CDS) could accelerate adoption of ED-initiated BUP into routine emergency care.

Objective: This study aimed to design and formatively evaluate a user-centered decision support tool for ED initiation of BUP for patients with OUD.

Methods: User-centered design with iterative prototype development was used. Initial observations and interviews identified workflows and information needs. The design team and key stakeholders reviewed prototype designs to ensure accuracy. A total of 5 prototypes were evaluated and iteratively refined based on input from 26 attending and resident physicians.

Results: Early feedback identified concerns with the initial CDS design: an alert with several screens. The timing of the alert led to quick dismissal without using the tool. User feedback on subsequent iterations informed the development of a flexible tool to support clinicians with varied levels of experience with the intervention by providing both one-click options for direct activation of care pathways and user-activated support for critical decision points. The final design resolved challenging navigation issues through targeted placement, color, and design of the decision support modules and care pathways. In final testing, users expressed that the tool could be easily learned without training and was reasonable for use during routine emergency care.

Conclusions: A user-centered design process helped designers to better understand users' needs for a Web-based clinical decision tool to support ED initiation of BUP for OUD. The process identified varying needs across user experience and familiarity with the protocol, leading to a flexible design supporting both direct care pathways and user-initiated decision support.

(JMIR Hum Factors 2019;6(1):e13121) doi: 10.2196/13121 


\section{KEYWORDS}

user-centered design; decision support systems, clinical; opioid-related disorders; opiate substitution treatment; health information technology

\section{Introduction}

\section{Background}

Opioid use disorder (OUD) is an escalating public health crisis that has impacted all regions of the United States and represents a substantial portion of emergency department (ED) visits each year. An estimated 2.1 million people in the United States have OUD [1] and 275 million people have OUD worldwide [2]. More than 33,000 opioid-related deaths occur annually in the United States and 118,000 opioid-related deaths occur annually worldwide [3]. From 2016 to 2017, EDs experienced a 30\% increase in visits for opioid overdose [4]. As the primary source of care for many people with OUD, the ED offers an important opportunity to engage patients receiving care for acute and comorbid conditions related to opioid use.

Buprenorphine (BUP), a partial opioid agonist often combined with an opioid antagonist, is a proven effective treatment for OUD that decreases mortality, withdrawal symptoms, craving, and opioid use [5-7]. Initiating BUP in the ED doubles the rate of addiction treatment engagement in ED patients with OUD [8]. However, ED-initiated BUP has not yet been adopted in most hospitals $[9,10]$. This delay in adoption of evidence-based practice is not unique-on average, it takes 17 years from discovery to the adoption of evidence-based practices into routine care $[11,12]$.

Clinical decision support (CDS), computerized systems that offer patient-specific assessments or recommendations to clinicians, represents one approach to facilitating and accelerating the implementation process [13,14]. A 2011 review of randomized controlled trials investigating CDS guidance for drug therapy showed that CDS improved care in 64\% (37/59) of studies [15]. In a broad review of CDS designed to address a range of care processes, meta-analysis favored the use of CDS for supporting clinician treatment orders (odds ratio 1.57, 95\% CI 1.35-1.82). Large studies have shown CDS implementation in the ED to have supported the adoption of evidence-based practices for computed tomography imaging use [16,17].

However, CDS faces its own challenges, including unintended consequences such as alert fatigue and increased cognitive load [18-22]. CDS design principles support careful consideration of the sociotechnical environment and delivery of the right information, to the right person, in the right format, and at the right time in clinical workflow to optimize medical decision making [23-26].

Across the fields of technology and human-computer interaction, building usable systems has been found to be essential to improve efficiency and reduce errors [27]. International Organization for Standardization standards for user-centered design outline the process by which technological design can incorporate context and organizational requirements to produce and evaluate solutions [28]. The engagement of end users (the people who will be using the technology) throughout the process is critical to anticipate and avoid pitfalls of new information technology such as increased cognitive load and lack of user engagement [26]. Specifically, pragmatic approaches to usability evaluation are necessary to rapidly design, iterate, and test health care information systems [29].

\section{Objectives}

Our objective was to develop a pragmatic, user-centered CDS for ED-initiated BUP and referral to treatment for patients with OUD. The user-centered design process for the development of this tool is described here. We developed this CDS specifically for the purposes of a planned multisystem pragmatic trial to study the effectiveness of user-centered CDS on adoption rates of ED-initiated BUP [30].

\section{Methods}

\section{Clinical Context and Population}

From March to July 2018, we utilized a multiphase, user-centered design methodology for the formative design, development, and evaluation of the EMergency department-initiated Buprenorphine for opioid usE Disorder (EMBED) CDS intervention. Primary phases in this method included (1) needs assessment, (2) initial prototype design, (3) iterative design feedback, and (4) final prototype testing. Formative feedback sessions were approved by our institution's institutional review board; given the minimal risk of the study and a protocol that did not involve the collection of participants' private information, all participants gave verbal consent for participation.

Eligible participants included ED clinicians and key stakeholders (including administrative and information technology leaders and ED addiction counselors) from an urban academic level I trauma center with 103,000 patient visits per year. Recruitment for user feedback sessions focused specifically on attending physicians and residents in the second, third, or fourth year of postgraduate medical training. During a 4-month period from March to June 2018, a total of 26 unique participants offered feedback during iterative design, including 14 through informal sessions and 12 through formal user feedback sessions. In addition, 6 participants offered feedback on multiple versions of the design. Informal sessions were conducted in the ED or private administrative offices and lasted 10 to $30 \mathrm{~min}$. Formal sessions were conducted in the Yale Center for Medical Simulation and were approximately $45 \mathrm{~min}$ in length. Formal user design sessions were conducted in parallel with both attending and resident physicians by a human factors researcher (JR).

\section{Pragmatic Approach}

Given our goal to rapidly increase adoption rates of ED-initiated BUP for a subsequent pragmatic trial, we elected to take a pragmatic approach to formative usability evaluation, as described by Mann et al [29]. This approach included rapid iterative design and testing cycles to provide user feedback and 
input on prototype design iterations. All sessions of user testing included direct observation, think aloud, and observational note-taking. In addition, notes were reviewed with each participant at the end of each session to ensure completeness. Pragmatic data analysis was performed by the design team during weekly meetings to debrief and summarize findings and to determine the design, functionality, and interface changes to make based on these findings. Termination was based on consensus, cost, and time constraints, as opposed to thematic saturation [29]. To minimize the assessment burden, we did not capture demographic data such as age, gender, race, or ethnicity (other than professional role) for the participants in the study [31].

\section{Phase 1: Needs Assessment}

The initial phase of design consisted of a focused discussion with key content and context experts as well as 3 ethnographic observation sessions of 2 to 5 hours in length. In the first 2 observations, the lead designer (MM) shadowed attending physicians in the ED. The third observation period focused on the processes of registration and the administration of patient flow through the waiting room and ED. Five 1-hour, individual interviews were then conducted with an ED drug and alcohol program counselor, a drug and alcohol treatment coordinator, an attending physician, and a resident. Interviews captured additional detail on workflow, roles, and user information needs.

\section{Phase 2: Initial Prototype Design}

After identifying potential users and their information needs, an initial low-fidelity prototype was designed. This prototype focused on key components necessary for implementing the ED-initiated BUP protocol, including modules to evaluate patients for OUD based on the Diagnostic and Statistical Manual of Mental Disorders, 5th Edition (DSM-5) criteria [32] and for opioid withdrawal severity using the Clinical Opioid Withdrawal Scale (COWS); the protocol for initiating BUP in the ED; and the steps necessary for referring patients for continued medication for OUD $[33,34]$. The initial prototype design was then reviewed by the design team as well as a subject matter expert on ED management of substance use disorder (GD) and a targeted sample of attending physicians and administrative leaders from the department. The goal of the initial prototype design phase was to establish the components necessary for the decision support tool and workflow. Questions identified during the initial review were addressed at this stage of design before moving forward to iterative design feedback sessions.

\section{Phase 3: Iterative Design Feedback and Prototype Revision}

With the initial static design complete, an interactive prototype was built in InVision (InVision, New York, NY). This prototype provided users with an interactive navigation and functionality experience. Feedback was gathered both through informal review and through formal user design sessions. Informal review included the distribution of electronic or print versions of all screens in the design to both attending and resident physicians. After verbal consent was obtained, each participant was oriented to the session format and read a case (Multimedia Appendix 1) of a patient presenting to the ED for treatment following an opioid overdose. Users were then given an electronic version of the CDS and asked to talk through how they would proceed. If participants did not initially mention the use of the tool, they were prompted to think about how and when they would expect to access the tool in this patient encounter. Participants were asked to think aloud describing how they expected to interact with the tool and were prompted for their initial reactions to it [35]. At the conclusion of each session, participants were asked to provide their overall impression of the tool's content and format as well as suggestions to make the tool easier to use and to increase the likelihood of incorporating it into their practice. All data were entered in a design log identifying the user need, recommendation, and changes resulting from those recommendations. Recommendations were reviewed by the design team weekly to determine how they should inform design revisions. After each iteration, additional feedback sessions were conducted to gather additional data and further refine design.

\section{Phase 4: Final Prototype Testing}

Final testing of the interactive InVision prototype consisted of formal user feedback sessions that proceeded until the design team reached a consensus that the prototype would exceed all users' needs $80 \%$ of the time based on the $80 / 20$ rule [36]. These sessions followed the format of the formal iterative design feedback sessions (detailed above in Phase 3). Participants included both resident and attending physicians with a wide range of experience with the ED-initiated BUP. Sampling was deliberate to include both participants from earlier iteration sessions as well as new participants naïve to the user design.

\section{Results}

\section{Phase 1 (Needs Assessments) and Phase 2 (Initial Prototype Design)}

Overall, 4 key topics for design were identified in Phase 1 (Table 1). These initial areas of concentration included appropriate patient identification, defining potential users of the decision support tool, avoiding workflow disruptions, CDS steps, and supporting user understanding of the treatment process. Attending physicians were expected to be the target system users, yet early observations and feedback suggested parts of the decision process might be completed by other members of the care team, such as medical students, residents, or nurses. This broadened view of system users, with varying clinical roles and experience, became an ongoing design challenge driving decisions of how and when to present support.

Activation of CDS tools was an early feedback topic. The initial design was an Epic (Epic Systems, Verona, WI) best practice alert (BPA; Figure 1) triggering a pop-up window when a patient was identified as potentially having OUD. However, users disliked the pop-up alert format as it could easily be dismissed if triggered at the wrong time in the clinical workflow, potentially causing a missed opportunity to support the intervention.

A second area of concern for users (throughout all iterations) was avoiding workflow disruptions; they preferred that the tool take no longer than 2 to $5 \mathrm{~min}$ to use. Similarly, users 
highlighted the need for system flexibility to accommodate for the user's experience level by allowing for decision support as needed as well as a direct care pathway selection with less support for more experienced clinicians.

Table 1. Needs assessment at baseline and ethnographic observation results.

\begin{tabular}{|c|c|}
\hline Needs/topics & How they were expressed \\
\hline \multirow[t]{4}{*}{ Appropriate patient identification } & Is it possible to have nurses identify patients with OUD ${ }^{\mathrm{a}}$ ? \\
\hline & Need to properly explain COWS ${ }^{b}$ to patients, who may understand it as "dope sick" \\
\hline & Can discharge instructions for opioid abuse be a trigger to activate $\operatorname{CDS}^{\mathrm{c}}$ ? \\
\hline & $\begin{array}{l}\text { There needs to be advanced search terms to trigger the CDS system-BPAs }{ }^{\mathrm{d}} \text { should not be the common denom- } \\
\text { inator for analysis }\end{array}$ \\
\hline \multirow[t]{3}{*}{ Avoiding workflow disruptions } & Avoid BPAs. They are intrusive and are rarely acted upon \\
\hline & Sometimes, physicians do leave electronic health record to access MDCalc or clinical resources websites \\
\hline & $\begin{array}{l}\text { Attending physicians usually do not have time for decision support. Better to tailor this toward residents and } \\
\text { nurses }\end{array}$ \\
\hline \multirow[t]{2}{*}{ Streamlining CDS steps } & $\begin{array}{l}\text { Integrate COWS into the } \mathrm{H} \& \mathrm{P}^{\mathrm{e}} \text { template, with integrated decision support and order sets to determine the need } \\
\text { for } \mathrm{BUP}^{\mathrm{f}}\end{array}$ \\
\hline & $\begin{array}{l}\text { If a user is initiated for OUD diagnosis, then workflow should be streamlined and skip through the diagnostic } \\
\text { criteria for OUD and go straight to treatment decision support }\end{array}$ \\
\hline \multirow[t]{6}{*}{ Understanding treatment process } & Should patients be given a $4 \mathrm{mg}$ or an $8 \mathrm{mg}$ dosage? \\
\hline & Need to have a short SBIRT ${ }^{\mathrm{g}}$ included in CDS to assess patient willingness to begin treatment \\
\hline & This is not the responsibility of our department but rather the substance abuse program \\
\hline & $\begin{array}{l}\text { Patients are rarely in the right range of withdrawal to prescribe BUP. Need to have a system to allow them to } \\
\text { return at an appropriate time to the } \mathrm{ED}^{\mathrm{h}}\end{array}$ \\
\hline & Some patients may have a preference for suboxone versus methadone \\
\hline & Should we have patients return to the ED for follow-up post BUP administration, using the 72-hour rule? \\
\hline
\end{tabular}

${ }^{\mathrm{a} O U D:}$ opioid use disorder.

${ }^{\mathrm{b}}$ COWS: Clinical Opioid Withdrawal Scale.

${ }^{\mathrm{c}} \mathrm{CDS}$ : clinical decision support.

${ }^{\mathrm{d}}$ BPA: best practice alert.

${ }^{\mathrm{e}} \mathrm{H} \&$ P: history and physical.

${ }^{f}$ BUP: buprenorphine.

${ }^{\mathrm{g}}$ SBIRT: Screening, Brief Intervention, and Referral to Treatment.

${ }^{\mathrm{h}} \mathrm{ED}$ : emergency department. 
Figure 1. Initial prototype user interface mockup as Epic best practice alert.

Best Practice Alert
Belecting 'Yes' to both questions will begin treatment protocol
Les

Figure 2. Second prototype user interface with optional decision support. DSM: Diagnostic and Statistical Manual for Mental Disorders; OUD: opioid use disorder.

\section{Buprenorphine Initiation Process}

Buprenorphine can be adminstered in patients who meet the following criteria:

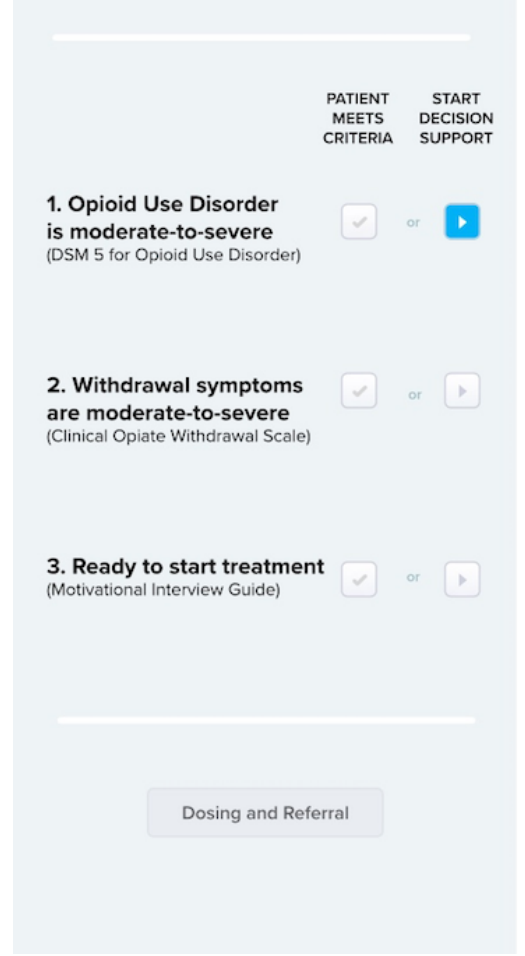

These needs informed the development of the first prototype that incorporated existing paper forms into 1 process. This first iteration presented step-by-step guidance through 6 sequential screens (Figure 1 includes the first slide): introductory BPA, DSM checklist for diagnosing OUD, COWS withdrawal assessment, motivational interview prompts, treatment options, and a referral form. Each step was delegated to a single screen to emphasize the discrete steps in a streamlined workflow.

\section{DSM - 5 CRITERIA FOR OPIOID USE DISORDER}

A patient that answers 'YES' to three or more of the following questions has moderate-to-severe OUD

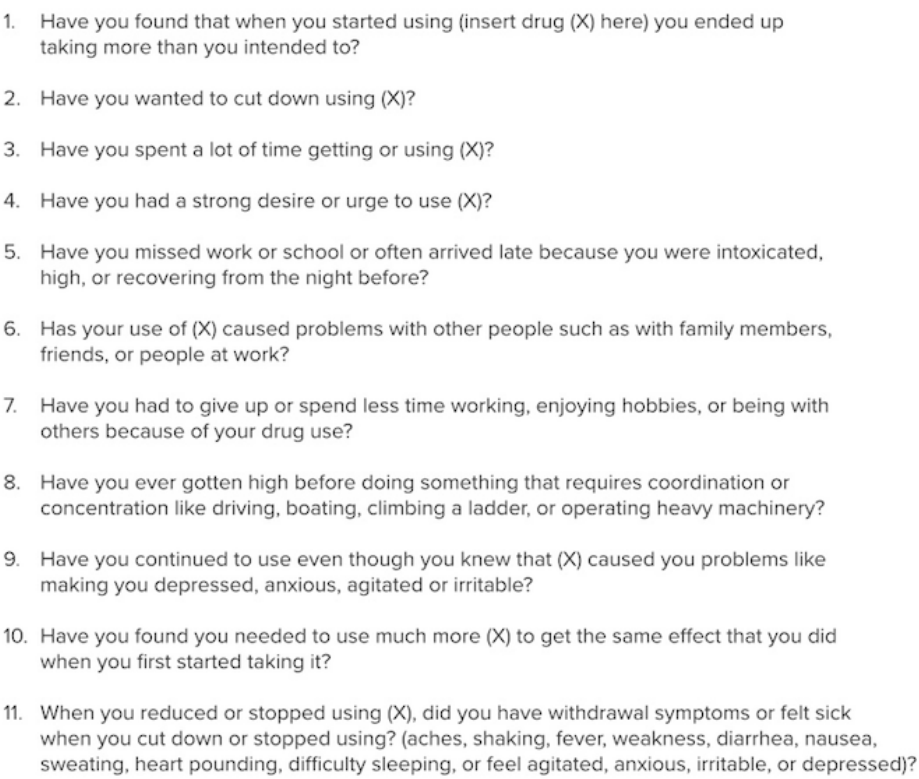

6. Has your use of $(X)$ caused problems with other people such as with family members, friends, or people at work?

Have you had to give up or spend less time working, enjoying hobbies, or being with others because of your drug use?

8. Have you ever gotten high before doing something that requires coordination or concentration like driving, boating, climbing a ladder, or operating heavy machinery?

Have you continued to use even though you knew that $(X)$ caused you problems like making you depressed, anxious, agitated or irritable?

D. Have you found you needed to use much more $(X)$ to get the same effect that you did when you first started taking it?

11. When you reduced or stopped using $(\mathrm{X})$, did you have withdrawal symptoms or felt sick when you cut down or stopped using? (aches, shaking, fever, weakness, diarrhea, nausea sweating, heart pounding, difficulty sleeping, or feel agitated, anxious, irritable, or depressed)?

\section{Phase 3 (Iterative Design)}

We created 5 major prototypes based on ongoing feedback. Multimedia Appendix 2 documents feedback received from each version and how it was incorporated into the subsequent revision. Across all prototypes, feedback focused on 4 thematic needs: design changes, navigation, workflow integration, and treatment process.

Feedback on the initial prototype (described above) focused on streamlining the prototype. This feedback was used to inform the second prototype (Figure 2) with the goal of a user-initiated 
CDS (instead of a BPA trigger) that could be embedded within the electronic health record (EHR) and further streamline the information in the individual steps.

Users found that this second iteration still had too many steps and too much text. They expressed difficulty in locating the decision support elements. Specific suggestions included consolidating steps with more clarity in regard to navigating the treatment options by including a progress bar.

These suggestions led to a complete redesign of the CDS in prototype 3 (Figure 3 ). To improve clarity and consolidate steps, this version included all treatment options in a single table on 1 screen with a row for each treatment option. User feedback for this version focused on optimizing the design by changing fonts, reducing the amount of text, and labeling treatment options and the decision support tools appropriately.

This feedback informed the design of prototype 4 (Figure 4 ) in which treatment pathways were presented in columns (rather than rows) with 1-click treatment pathway selection at the bottom of each column. Buttons in the far left column provided access to modules for OUD diagnosis, withdrawal assessment, and motivation and assessment of patient readiness for treatment. Feedback for this version was mostly positive, with minor navigation concerns about where to start the tool and how to activate the decision support.

Figure 3. Third prototype user interface single-click care pathways. BUP: buprenorphine; COWS: Clinical Opioid Withdrawal Scale; DSM: Diagnostic and Statistical Manual of Mental Disorders; EHR: electronic health record; SL/PO: sublingual/by mouth.

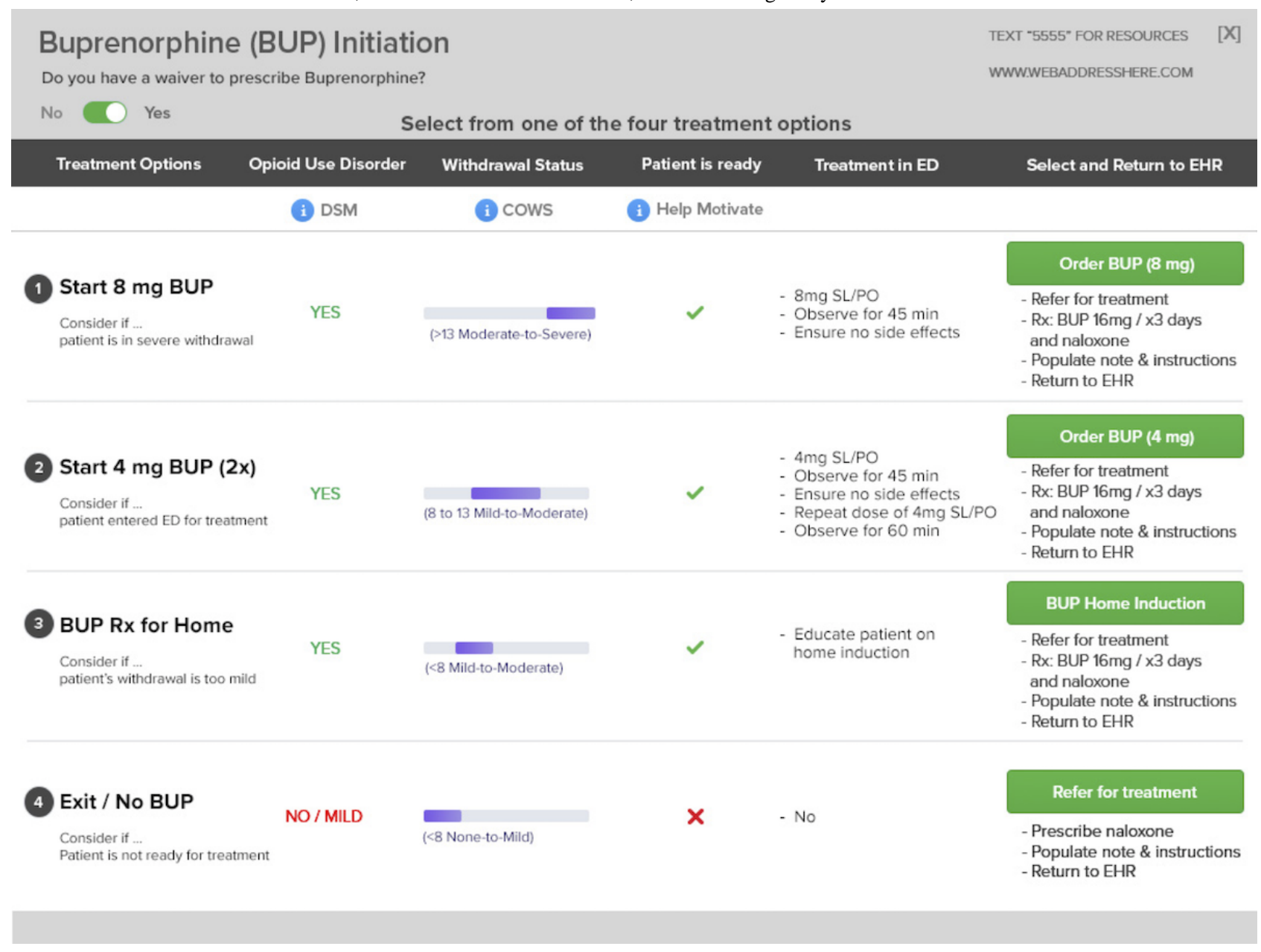


Figure 4. Fourth prototype user interface with care pathways to columns. BUP: buprenorphine; COWS: Clinical Opioid Withdrawal Scale; DSM: Diagnostic and Statistical Manual of Mental Disorders; SL/PO: sublingual/by mouth.

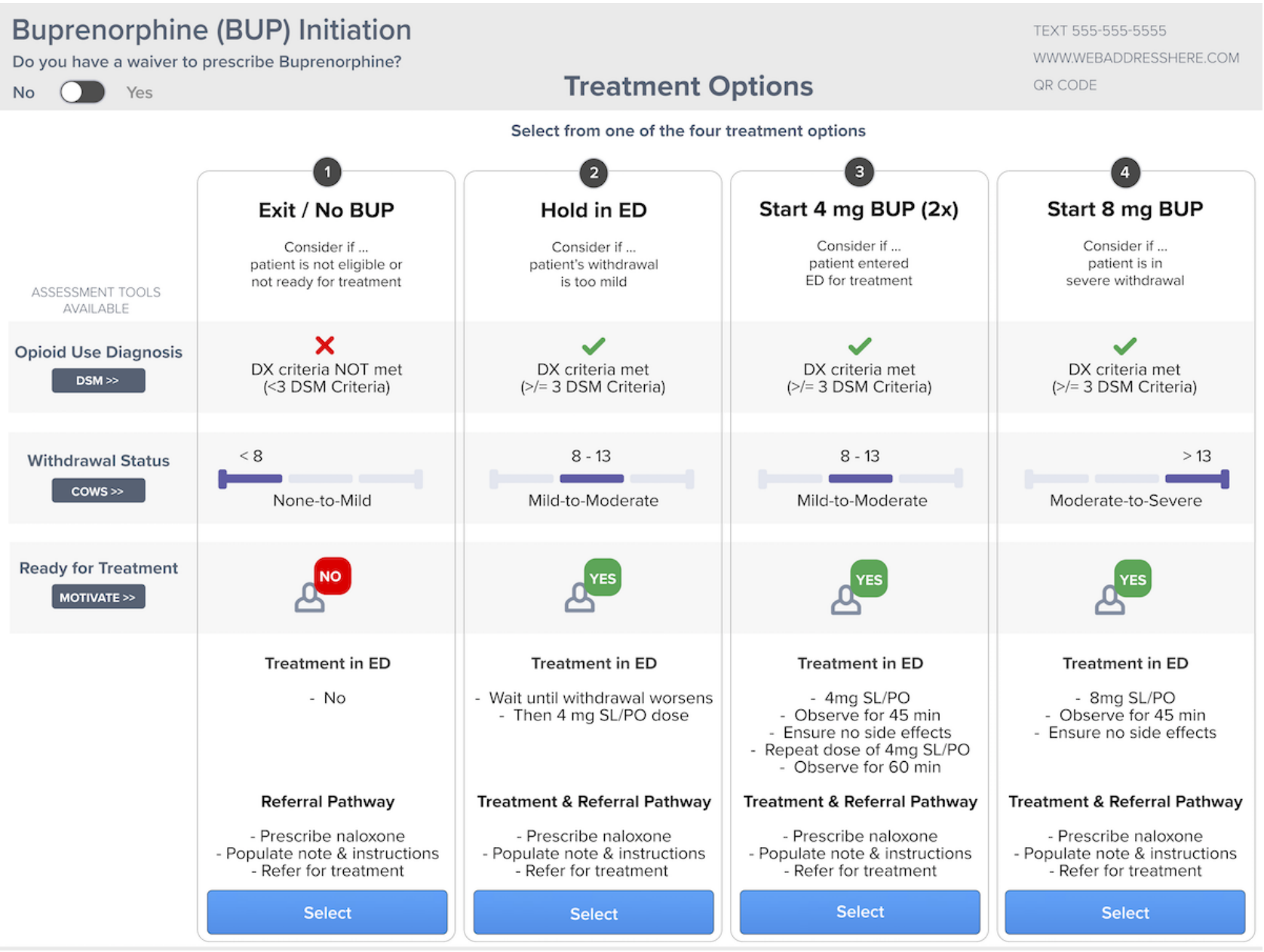

\section{Phase 4 (Final Prototype Testing)}

The final prototype design goal was an intuitive, simple layout offering flexibility for direct treatment or user-initiated decision support. In response to navigation concerns, nonessential text was removed, and decision support was presented with blue buttons in the far right column, following the horizontal path for the DSM, COWS, and motivational interview (Figure 5). Although feedback was generally positive for the simplified layout, users suggested that the direct care pathways needed clear delineation. These concerns were addressed in a final design change to outline each treatment column. During final testing, multiple users initially attempted to click in the middle of the main screen to select a care pathway, so care pathway activation buttons were changed to green to indicate the start of treatment. All participants at this stage thought that the system was easy to learn without training and reasonable for use in their routine emergency care practice. Figure 6 summarizes the needs assessment and general workflow of our intervention based on all phases of formative evaluation. 
Figure 5. Final prototype user interface with decision support moved to the right column. BUP: buprenorphine; COWS: Clinical Opioid Withdrawal Scale; DSM: Diagnostic and Statistical Manual of Mental Disorder; OUD: opioid use disorder.

\begin{tabular}{|c|c|c|}
\hline \multicolumn{2}{|c|}{ Buprenorphine (BUP) Initiation } & TEXT 555-555-5555 \\
\hline Do you have a waiv & & WWWWEBADDRESSHERE.COM \\
\hline No $\bigcirc$ Yes & Buprenorphine Treatment Options & QR CODE \\
\hline
\end{tabular}

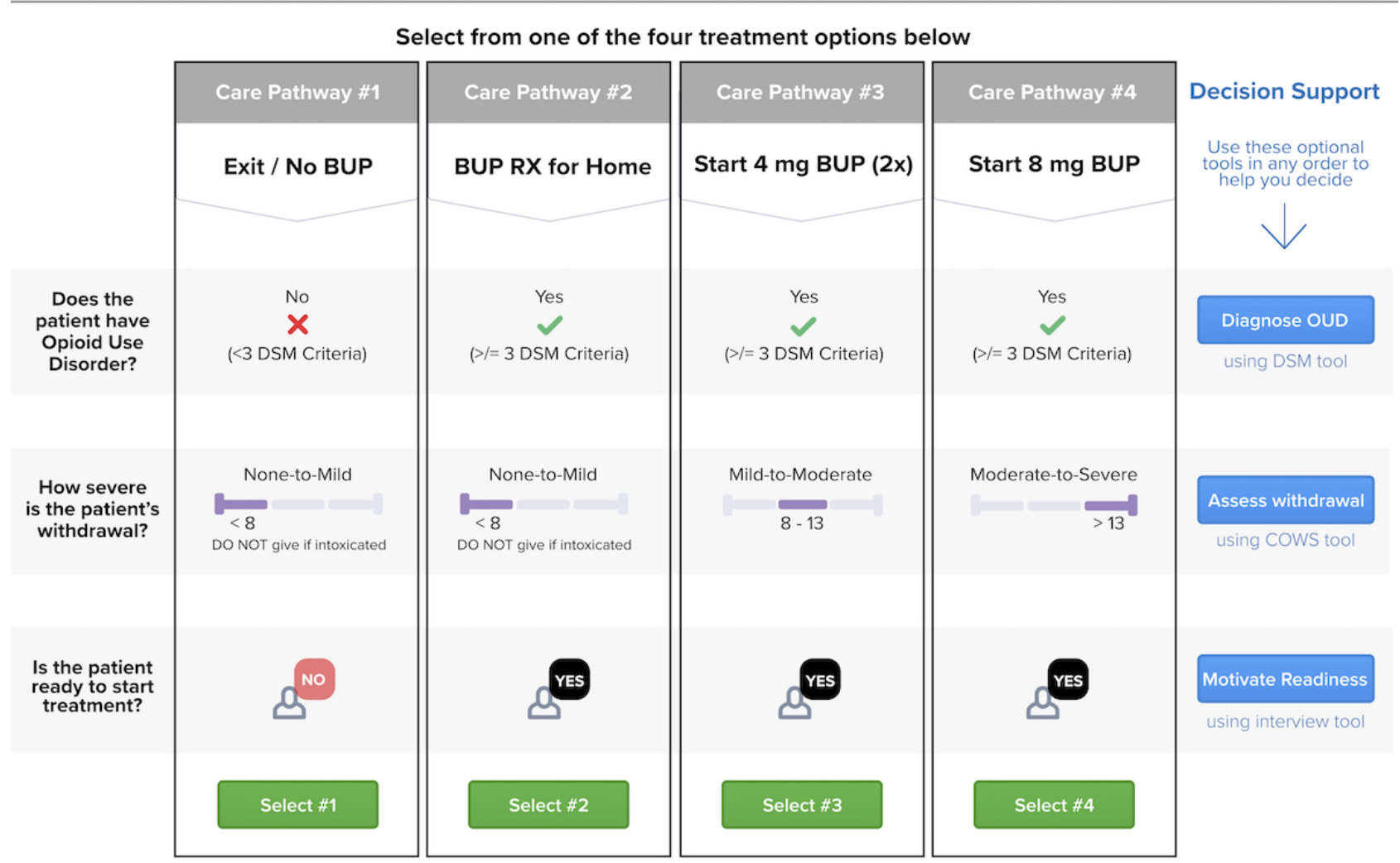

Figure 6. Final information technology workflow for user-centered clinical decision support intervention based on formative evaluation. ED: emergency department; EMBED: EMergency department-initiated Buprenorphine for opioid usE Disorder; EHR: electronic health record; OUD: opioid use disorder.

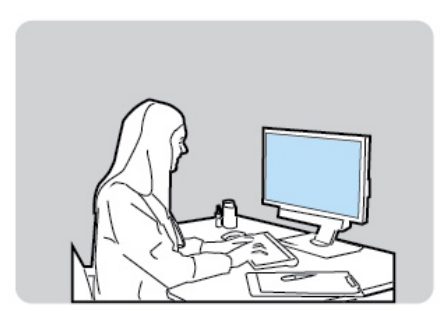

(1) ED clinicians will conduct their usual workflow within their current EHR environment.

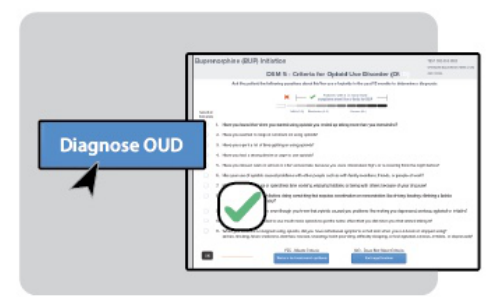

4 Other members of the care team can complete all or just some of the assessment tools, specifically for diagnosing OUD, scoring withdrawal symptoms, and determining a patient's readiness for treatment.

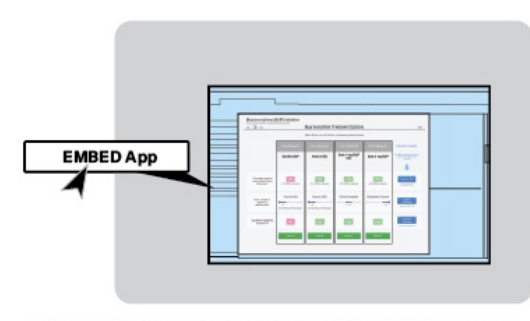

When the appropriate patient is identified, the clinician can click on the button to launch the "EMBED" decision can click on the button to launch the "EME
support app without leaving the EHR.
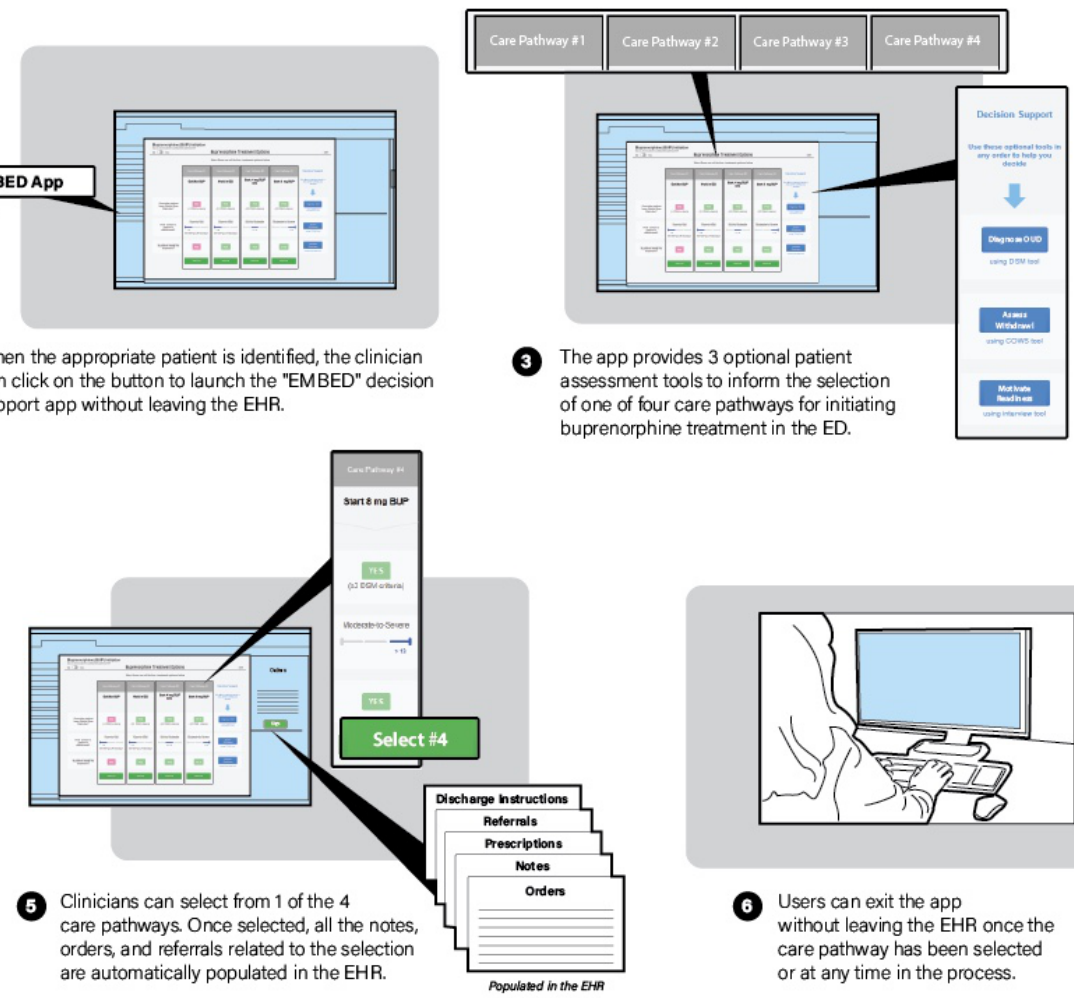

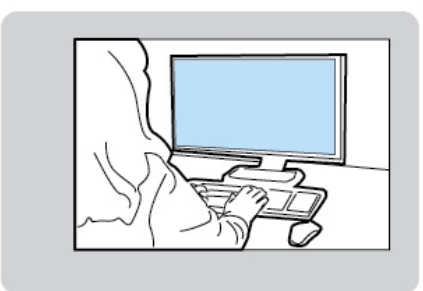

6 Users can exit the app without leaving the EHR once the care pathway has been selected or at any time in the process. 


\section{Discussion}

\section{Principal Findings}

We describe the iterative user-centered design process to develop a CDS for ED-initiated BUP. Across 4 phases with 5 major revisions and continuous iteration, we identified user needs for a flexible tool to support members of the care team who could be either experienced users or those new to ED-initiated BUP. Interactive feedback sessions identified key themes throughout the refinement process, including issues of navigation, overall design recommendations, considerations for workflow integration, and questions regarding the treatment protocol. Throughout the design process, how and where to activate decision support represented a key challenge. Early prototype versions provided step-by-step guidance through existing forms and processes. Requests for a more efficient and flexible tool resulted in an easy-to-read layout with options for direct clinical care activation or user-activated decision support. The challenge of simultaneously providing both a direct care pathway and flexible decision support led to a design with multiple navigation options. Adoption of key design principles included minimizing unnecessary text, utilizing a standard form and colors for buttons, and layout of information in meaningful pathways. We selected a final design once all user-identified concerns had been addressed and user feedback indicated the tool was meeting their anticipated needs.

\section{Strengths and Meaning of the Study}

This study supports the use of user-centered design. Through both formal and informal feedback, we captured user needs and input that would not be captured in traditional CDS design processes that lack a needs assessment or formative evaluation. Expert review of content ensured accuracy, whereas feedback from users with varying levels of experience highlighted the need for flexible support. Comments from expert users emphasized the need for an option to directly launch the desired care pathway. Although a direct care pathway provides flexibility for expert clinicians, novice clinicians emphasized the need for more structured decision support and clarity in the diagnostic and treatment processes. Specifically, less experienced users welcomed the detailed criteria for OUD diagnosis and withdrawal assessment, instructions for conducting a motivational interview, and clarity regarding BUP dosing. This contrast in user needs presented a design challenge that highlights the importance of sampling participants across the range of user experience levels with the protocol supported by the CDS. A deliberate sampling of participants from earlier iteration sessions as well as new participants provided confirmatory feedback on how recommended changes were incorporated into the design. Finally, design relied on both user comments and existing standards for layout and use of specific design features such as color.

\section{Limitations}

As the CDS supports a treatment pathway, the underlying workflow driving development was identified as clinician workflow. As such, clinicians were the primary users studied. Less focus on other members of the care team could represent a limitation-in particular, if the user population is broadened in implementation at the site where the design process was conducted or at other sites using the tool in the future. A number of users suggested a role for other nonclinician staff in identifying OUD patients in the ED. In particular, multiple clinicians mentioned that the COWS could be completed by a nurse. Therefore, the tool is designed with resources that can be used by or distributed to other members of the care team (eg, nurse, medical student, and addiction counselor). In this way, a nonclinician could still complete the diagnostic or withdrawal assessment, and the clinician could incorporate this assessment into their final care pathway selection.

Given the urgency of the opioid epidemic, we made a conscious decision to take a pragmatic approach to the design and formative evaluation of our intervention. Developing the CDS through a pragmatic approach instead of a traditional academic approach allowed for the rapid inclusion of user feedback in a shorter time frame [29]. We recognize that limitations to this approach exist, including the potential for additional data that could be captured in a deeper, more rigorous data analysis typical of the academic approach. With a traditional academic approach, data saturation anticipates capturing $100 \%$ of user feedback themes, whereas this pragmatic approach to development relies on capturing 80\% of critical issues [36]. However, we were willing to accept this trade-off to achieve the aim of the subsequent trial to accelerate getting this life-saving treatment into routine emergency care.

This work represents the initial phase of a larger project for the development, implementation, and testing of the effectiveness of the CDS developed here. Design and user feedback sessions were conducted at a single site, though implementation will include multiple sites and could potentially interface with other vendors' EHRs. Having a limited group of users engaged in design is practical and not unique to our work. However, we recognize that this introduces the potential for design features supporting local norms and processes that may not be generalizable. To mitigate this potential limitation, we sought feedback throughout the design process from external collaborators as well as guidance from a subject matter expert on ED management of substance use disorder.

\section{Comparison With Prior Work}

Given the devastating toll of the opioid epidemic, this user-centered CDS was developed to give clinicians the tools necessary to engage more people suffering from OUD in effective treatment at a time when they may be particularly open to it [8-10]. However, this intervention may be challenging to disseminate for several reasons: (1) it implements a multistep practice that is not familiar to clinicians; (2) ED clinicians are unlikely to see immediate effects of their efforts; (3) the targeted patient population is often perceived to be difficult to work with; and (4) the legal status of BUP for OUD is complicated, requiring a special waiver to prescribe for home use, but no waiver required if the treatment is administered onsite for no more than 72 hours $[37,38]$.

Given these challenges to adoption, we perceived an opportunity to increase the likelihood of success by employing user-centered design to create the EMBED CDS intervention. Emerging literature supports this approach [39-42]. For example, Thursky 
and Mahemoff have incorporated participatory design methods to create antibiotic CDS for physicians in intensive care units [39]. Kilsdonk et al have employed user-centered decision support to create a tool that improved the speed and accuracy of clinician's identification of appropriate screening procedures for childhood cancer survivors, relative to the use of a paper guideline [40]. Plaisance et al have used a process similar to our study to design a CDS for cardiopulmonary resuscitation in the intensive care unit [42]. We have also previously employed a user-centered design in developing CDS for patients with head injury in the ED [41].

Notably, these studies have shown that including user feedback in the design phase leads to greater effectiveness and efficiency and, ultimately, to a sense of physician ownership of the CDS, which increases its immediate uptake and continued use. They have also highlighted rapid-cycle prototyping with user engagement throughout a design process [42]. Similar to previous reports in this area, we found that different groups of users expressed different needs for the tool. We approached this challenge through a design approach that balanced the goals, priorities, and information processing needs of both novice and expert users. This resulted in a tool that could support multiple types of users and their preferred workflows. We demonstrate how a single tool can be designed with the flexibility to meet multiple users' work processes and information processing needs. Designing for the human requires an understanding of workflows, information needs, priorities, and preferences; user-centered design captures this through user engagement across the design and development life cycle [26-28].

\section{Conclusions}

This work describes the design and formative evaluation of a user-centered CDS for ED-initiated BUP. We add to the expanding literature on the design of user-centered CDS tools by describing the process and challenges of designing a flexible tool that supports both novice and expert clinicians in identifying appropriate patients and appropriate care pathways. Future work will include summative usability evaluation and pilot testing of the intervention to further optimize the tool for wide-scale implementation within existing ED workflows in a large pragmatic clinical trial across multiple health care systems. The aim of this subsequent pragmatic trial is to increase adoption of ED-initiated BUP for people suffering from OUD, thereby decreasing morbidity and mortality associated with opioid addiction. Users will also inform pilot implementation in a series of focus groups. Although early engagement of users supports the design process, we anticipate continued support of potential users will be equally important across the project life cycle.

\section{Acknowledgments}

Research reported in this publication was supported within the NIH Health Care Systems Research Collaboratory by a cooperative agreement (UG3DA047003) from the National Institute on Drug Abuse of the NIH. This work also received logistical and technical support from the NIH Collaboratory Coordinating Center (U24AT009676). The content is solely the responsibility of the authors and does not necessarily represent the official views of the National Institutes of Health. The funders had no role in study design, data collection and analysis, decision to publish, or preparation of the manuscript.

\section{Authors' Contributions}

ERM, JMR, MM, and GD conceived and designed the work. All authors substantially contributed to the acquisition, analysis, and interpretation of the study data. JMR and OMA drafted the initial manuscript. All authors edited and approved the final version submitted for publication. ERM takes responsibility for the study as a whole.

\section{Conflicts of Interest}

None declared.

\section{Multimedia Appendix 1}

User-centered design script.

[PDF File (Adobe PDF File), 179KB-Multimedia Appendix 1]

\section{Multimedia Appendix 2}

Design iterations, feedback, and solutions.

[PDF File (Adobe PDF File), 168KB-Multimedia Appendix 2]

\section{References}

1. Center for Behavioral Health Statistics and Quality. Substance Abuse and Mental Health Services Administration. 2016. Key substance use and mental health indicators in the United States: Results from the 2015 National Survey on Drug Use and Health (HHS Publication No URL: http://www.samhsa.gov/data/ [accessed 2018-12-15] [WebCite Cache ID 74hC6qmq5]

2. World Health Organization. Information sheet on opioid overdose URL: https://www.who.int/substance abuse/ information-sheet/en/ [accessed 2019-02-01] [WebCite Cache ID 75rorCStG] 
3. Seth P, Scholl L, Rudd RA, Bacon S. Overdose deaths involving opioids, cocaine, and psychostimulants-United States, 2015-2016. MMWR Morb Mortal Wkly Rep 2018 Mar 30;67(12):349-358 [FREE Full text] [doi: 10.15585/mmwr.mm6712a1] [Medline: 29596405]

4. Vivolo-Kantor AM, Seth P, Gladden RM, Mattson CL, Baldwin GT, Kite-Powell A, et al. Vital signs: trends in emergency department visits for suspected opioid overdoses-United States, July 2016-September 2017. MMWR Morb Mortal Wkly Rep 2018 Mar 9;67(9):279-285 [FREE Full text] [doi: 10.15585/mmwr.mm6709e1] [Medline: 29518069]

5. Kakko J, Svanborg KD, Kreek MJ, Heilig M. 1-year retention and social function after buprenorphine-assisted relapse prevention treatment for heroin dependence in Sweden: a randomised, placebo-controlled trial. Lancet 2003 Feb 22;361(9358):662-668. [doi: 10.1016/S0140-6736(03)12600-1] [Medline: 12606177]

6. Mattick RP, Breen C, Kimber J, Davoli M. Buprenorphine maintenance versus placebo or methadone maintenance for opioid dependence. Cochrane Database Syst Rev 2014 Feb 6(2):CD002207. [doi: 10.1002/14651858.CD002207.pub4] [Medline: 24500948]

7. Larochelle MR, Liebschutz JM, Zhang F, Ross-Degnan D, Wharam JF. Opioid prescribing after nonfatal overdose and association with repeated overdose. Ann Intern Med 2016 Dec 6;165(5):376-377. [doi: 10.7326/L16-0168] [Medline: 27595218]

8. D'Onofrio G, O'Connor PG, Pantalon M, Chawarski M, Busch S, Owens P, et al. Emergency department-initiated buprenorphine/naloxone treatment for opioid dependence: a randomized clinical trial. J Am Med Assoc 2015 Apr 28;313(16):1636-1644 [FREE Full text] [doi: 10.1001/jama.2015.3474] [Medline: 25919527]

9. Duber HC, Barata IA, Cioè-Peña E, Liang SY, Ketcham E, Macias-Konstantopoulos W, et al. Identification, management, and transition of care for patients with opioid use disorder in the emergency department. Ann Emerg Med 2018 Oct;72(4):420-431. [doi: 10.1016/j.annemergmed.2018.04.007] [Medline: 29880438]

10. Martin A, Mitchell A, Wakeman S, White B, Raja A. Emergency department treatment of opioid addiction: an opportunity to lead. Acad Emerg Med 2018 May;25(5):601-604. [doi: 10.1111/acem.13367] [Medline: 29266577]

11. Balas EA, Boren SA. Managing clinical knowledge for health care improvement. Yearb Med Inform 2000(1):65-70. [Medline: 27699347]

12. Morris ZS, Wooding S, Grant J. The answer is 17 years, what is the question: understanding time lags in translational research. J R Soc Med 2011 Dec;104(12):510-520 [FREE Full text] [doi: 10.1258/jrsm.2011.110180] [Medline: 22179294]

13. Kawamoto K, Houlihan CA, Balas EA, Lobach DF. Improving clinical practice using clinical decision support systems: a systematic review of trials to identify features critical to success. Br Med J 2005 Apr 2;330(7494):765 [FREE Full text] [doi: $10.1136 / \mathrm{bmj} .38398 .500764 .8 \mathrm{~F}$ ] [Medline: 15767266 ]

14. Garg AX, Adhikari NKJ, McDonald H, Rosas-Arellano MP, Devereaux PJ, Beyene J, et al. Effects of computerized clinical decision support systems on practitioner performance and patient outcomes: a systematic review. J Am Med Assoc 2005 Mar 9;293(10):1223-1238. [doi: 10.1001/jama.293.10.1223] [Medline: 15755945$]$

15. Hemens BJ, Holbrook A, Tonkin M, Mackay JA, Weise-Kelly L, Navarro T, CCDSS Systematic Review Team. Computerized clinical decision support systems for drug prescribing and management: a decision-maker-researcher partnership systematic review. Implement Sci 2011 Aug 3;6:89 [FREE Full text] [doi: 10.1186/1748-5908-6-89] [Medline: 21824383]

16. Raja AS, Ip IK, Prevedello LM, Sodickson AD, Farkas C, Zane RD, et al. Effect of computerized clinical decision support on the use and yield of CT pulmonary angiography in the emergency department. Radiology 2012 Feb;262(2):468-474 [FREE Full text] [doi: 10.1148/radiol.11110951] [Medline: 22187633]

17. Sharp AL, Huang BZ, Tang T, Shen E, Melnick ER, Venkatesh AK, et al. Implementation of the Canadian CT Head Rule and its association with use of computed tomography among patients with head injury. Ann Emerg Med 2018 Jan;71(1):54-63.e2 [FREE Full text] [doi: 10.1016/j.annemergmed.2017.06.022] [Medline: 28739290]

18. Sittig DF, Wright A, Osheroff JA, Middleton B, Teich JM, Ash JS, et al. Grand challenges in clinical decision support. J Biomed Inform 2008 Apr;41(2):387-392 [FREE Full text] [doi: 10.1016/j.jbi.2007.09.003] [Medline: 18029232]

19. Ash JS, Berg M, Coiera E. Some unintended consequences of information technology in health care: the nature of patient care information system-related errors. J Am Med Inform Assoc 2004 Apr;11(2):104-112 [FREE Full text] [doi: 10.1197/jamia.M1471] [Medline: 14633936]

20. Ash JS, Sittig DF, Campbell EM, Guappone KP, Dykstra RH. Some unintended consequences of clinical decision support systems. AMIA Annu Symp Proc 2007:26-30 [FREE Full text] [Medline: 18693791]

21. Levin S, France DJ, Hemphill R, Jones I, Chen KY, Rickard D, et al. Tracking workload in the emergency department. Hum Factors 2006;48(3):526-539. [doi: 10.1518/001872006778606903] [Medline: 17063967]

22. Melnick ER, Nielson JA, Finnell JT, Bullard MJ, Cantrill SV, Cochrane DG, et al. Delphi consensus on the feasibility of translating the ACEP clinical policies into computerized clinical decision support. Ann Emerg Med 2010 Oct;56(4):317-320. [doi: 10.1016/j.annemergmed.2010.03.006] [Medline: 20363531]

23. Sirajuddin AM, Osheroff JA, Sittig DF, Chuo J, Velasco F, Collins DA. Implementation pearls from a new guidebook on improving medication use and outcomes with clinical decision support. Effective CDS is essential for addressing healthcare performance improvement imperatives. J Healthc Inf Manag 2009;23(4):38-45 [FREE Full text] [Medline: 19894486] 
24. Phansalkar S, Edworthy J, Hellier E, Seger DL, Schedlbauer A, Avery AJ, et al. A review of human factors principles for the design and implementation of medication safety alerts in clinical information systems. J Am Med Inform Assoc 2010;17(5):493-501 [FREE Full text] [doi: 10.1136/jamia.2010.005264] [Medline: 20819851]

25. Horsky J, Phansalkar S, Desai A, Bell D, Middleton B. Design of decision support interventions for medication prescribing. Int J Med Inform 2013 Jun;82(6):492-503. [doi: 10.1016/j.ijmedinf.2013.02.003] [Medline: 23490305]

26. Gellert G, Webster S, Gillean J, Melnick E, Kanzaria H. Should US doctors embrace electronic health records? Br Med J 2017 Dec 24;356:j242. [doi: 10.1136/bmj.j242] [Medline: 28119282]

27. Maguire M. Methods to support human-centred design. Int J Hum Comput Stud 2001 Oct 1;55(4):587-634. [doi: 10.1006/ijhc.2001.0503]

28. International Organization for Standardization. 2015. ISO 9241-210:2010: Ergonomics of human-system interaction - Part 210: Human-centred design for interactive systems URL: https://www.iso.org/standard/52075.html [accessed 2018-12-16] [WebCite Cache ID 74hCVihfE]

29. Mann DM, Chokshi SK, Kushniruk A. Bridging the gap between academic research and pragmatic needs in usability: a hybrid approach to usability evaluation of health care information systems. JMIR Hum Factors 2018 Nov 28;5(4):e10721 [FREE Full text] [doi: 10.2196/10721] [Medline: $\underline{\text { 30487119] }}$

30. NIH Collaboratory Rethinking Clinical Trials. UG3 Project: Pragmatic Trial of User-Centered Clinical Decision Support to Implement EMergency Department-Initiated BuprenorphinE for Opioid Use Disorder (EMBED) URL: https://tinyurl. com/y35dz7b8/ [accessed 2019-02-01] [WebCite Cache ID 75rp6Sgio]

31. Bernstein SL, Rosner J, DeWitt M, Tetrault J, Hsiao AL, Dziura J, et al. Design and implementation of decision support for tobacco dependence treatment in an inpatient electronic medical record: a randomized trial. Transl Behav Med 2017 Dec;7(2):185-195 [FREE Full text] [doi: 10.1007/s13142-017-0470-8] [Medline: 28194729]

32. Bernstein E, Bernstein J, Weiner S, D’Onofrio G. Substance use disorders. In: Tintinalli's Emergency Medicine: A Comprehensive Study Guide. New York: Mcgraw-Hill; 2016.

33. American Psychiatric Association. Diagnostic and Statistical Manual of Mental Disorders (DSM-5®). Washington, DC: American Psychiatric Publishing; 2013.

34. Wesson D, Ling W. The Clinical Opiate Withdrawal Scale (COWS). J Psychoactive Drugs 2003;35(2):253-259. [doi: 10.1080/02791072.2003.10400007] [Medline: 12924748]

35. Li AC, Kannry JL, Kushniruk A, Chrimes D, McGinn TG, Edonyabo D, et al. Integrating usability testing and think-aloud protocol analysis with "near-live" clinical simulations in evaluating clinical decision support. Int J Med Inform 2012 Nov;81(11):761-772. [doi: 10.1016/j.ijmedinf.2012.02.009] [Medline: 22456088]

36. Usability.gov. 80/20 rule URL: https://www.usability.gov/what-and-why/glossary/8020-rule.html [accessed 2019-02-01] [WebCite Cache ID 75rpHWnL3]

37. Gawande A. New Yorker. Slow ideas URL: https://www.newyorker.com/magazine/2013/07/29/slow-ideas [accessed 2018-12-15] [WebCite Cache ID 74hCoI87F]

38. Dearing JW, Cox JG. Diffusion of innovations theory, principles, and practice. Health Aff 2018 Dec;37(2):183-190. [doi: 10.1377/hlthaff.2017.1104] [Medline: 29401011]

39. Thursky KA, Mahemoff M. User-centered design techniques for a computerised antibiotic decision support system in an intensive care unit. Int J Med Inform 2007 Oct;76(10):760-768. [doi: 10.1016/j.ijmedinf.2006.07.011] [Medline: 16950650]

40. Kilsdonk E, Peute LW, Riezebos RJ, Kremer LC, Jaspers MW. From an expert-driven paper guideline to a user-centred decision support system: a usability comparison study. Artif Intell Med 2013 Sep;59(1):5-13. [doi:

10.1016/j.artmed.2013.04.004] [Medline: 23684240]

41. Melnick ER, Hess EP, Guo G, Breslin M, Lopez K, Pavlo AJ, et al. Patient-centered decision support: formative usability evaluation of integrated clinical decision support with a patient decision aid for minor head injury in the emergency department. J Med Internet Res 2017 May 19;19(5):e174 [FREE Full text] [doi: 10.2196/jmir.7846] [Medline: 28526667]

42. Plaisance A, Witteman HO, LeBlanc A, Kryworuchko J, Heyland DK, Ebell MH, et al. Development of a decision aid for cardiopulmonary resuscitation and invasive mechanical ventilation in the intensive care unit employing user-centered design and a wiki platform for rapid prototyping. PLoS One 2018;13(2):e0191844 [FREE Full text] [doi: 10.1371/journal.pone.0191844] [Medline: 29447297]
Abbreviations
BPA: best practice alert
BUP: buprenorphine
CDS: clinical decision support
COWS: Clinical Opioid Withdrawal Scale
DSM: Diagnostic and Statistical Manual of Mental Disorders
ED: emergency department
EMBED: EMergency department-initiated Buprenorphine for opioid usE Disorder
OUD: opioid use disorder 


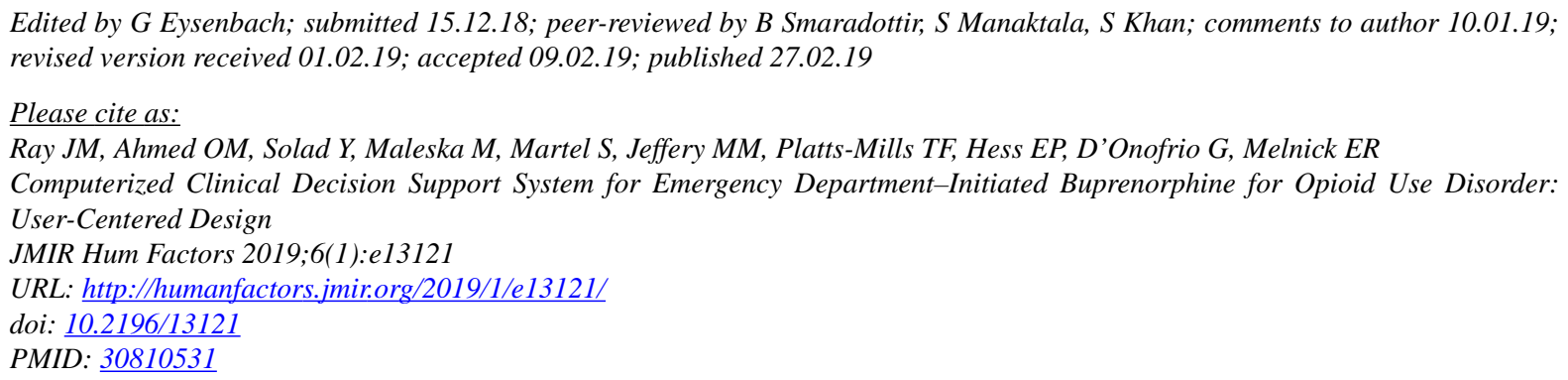

CJessica M Ray, Osama M Ahmed, Yauheni Solad, Matthew Maleska, Shara Martel, Molly M Jeffery, Timothy F Platts-Mills, Erik P Hess, Gail D'Onofrio, Edward R Melnick. Originally published in JMIR Human Factors (http://humanfactors.jmir.org), 27.02.2019. This is an open-access article distributed under the terms of the Creative Commons Attribution License (https://creativecommons.org/licenses/by/4.0/), which permits unrestricted use, distribution, and reproduction in any medium, provided the original work, first published in JMIR Human Factors, is properly cited. The complete bibliographic information, a link to the original publication on http://humanfactors.jmir.org, as well as this copyright and license information must be included. 